\title{
Using POD method in experiments with convection flow on the cube benchmark
}

\author{
Daniil Sergeev ${ }^{1,2^{*}}$, Alexander Kandaurov ${ }^{1,2}$ \\ ${ }^{1}$ Institute of applied physics RAS, Geophysical Research Division, 603950 Ulyanova st. 46, Nizhny Novgorod, Russia \\ ${ }^{2}$ Nizhny Novgorod State Technical University n.a. R.E. Alekseev, 603950 Minina st. 24, Nizhny Novgorod, Russia
}

\begin{abstract}
Investigations of the flow structure on the benchmark "cube" under conditions of natural convection caused by a vertical temperature gradient are performed using a low cost PIV-measuring system with continuous laser. The obtained velocity fields were processed with POD method to obtain typical structures (modes) in the flow. It is shown that the steady flow can be described by combination of the first three modes. It is demonstrated that only starting with the third mode there is a significant change in the spectra of the amplitude coefficients of the modes, the peak shifts to the region of higher frequencies. Thus accounting for this mode is essential for describing turbulent processes in the system and verification of numerical calculations. Comparison with the results of the LES simulation performed using the Ansys CFX package (taking into account the results of processing the experimental data by the POD method) demonstrated good agreement both in the structure of the modes and in their frequency spectra.
\end{abstract}

\section{Introduction}

For processes in power plants (including nuclear), the task of predicting temperature fields in non-isothermal flows is of current importance. Unsteady non-isothermal flows have a significant effect on the readings of temperature sensors, which are used to monitor the control of nuclear reactor facilities. In addition, temperature fluctuations occur in turbulent nonisothermal flows, which cause additional thermal cyclic loads on the equipment walls and in some cases significantly reduce the lifetime of individual equipment and the facility as a whole.

Experts from CFD4NRS (nuclear reactor safety working group) recommend an experiment on investigation of the natural convection of air inside a cubic cavity with a given temperature difference of opposite vertical walls for verifying experiments that model the conditions of natural convection. It should be noted that natural convection in closed cavities is a traditional object of basic research, attracting the attention of the diversity of its dynamic modes [1,2].

In this paper, it is proposed to consider the convection of water in a cubic cavity heated from below as such a reference problem (benchmark).

Natural convection in closed cavities is a basic object for modeling of various convective processes in engineering problems. The greatest number of works is devoted to the study of convection in rectangular cavities with horizontal temperature difference (heating and cooling on the side walls).

The situation with the vertical temperature difference is much more complicated, since in this case a whole set of large-scale convective currents is possible. The main studies of large-scale flows in closed volumes are performed for convection in cylindrical and cubic cavities. The first numerical calculations of convection in a cubic cavity with a vertical temperature difference were carried out for moderate values of the Rayleigh number $\left(3.5 \cdot 10^{3} \leq \mathrm{Ra} \leq 6 \cdot 10^{4}\right)[3-6]$. It was shown that even for small values of supercriticality in laminar regimes, the formation of flows of various types is possible.

In the context of the discussed applications, developed turbulent convection is of interest (with Rayleigh numbers $10^{8} \leq \mathrm{Ra} \leq 10^{13}$ and higher), but little research has been done on such regimes. This is due to the fact that the evolution of large-scale flows in turbulent regimes proceeds rather slowly, and, as experiments show, it takes dozens of hours to describe the time evolution of large-scale structures. Carrying out calculations at such characteristic times is a non-trivial task and requires careful selection of a numerical model and high performance of the computing system. Therefore, the verification procedure is very important here.

For the problem of verifying the results of numerical simulation, a representative comparison of the results of numerical calculation and experimental data is of the most importance. Comparison of snapshots or average flow patterns does not allow to sufficiently verify the results of numerical calculations. For verification, a comparison of the spectral characteristics of flows at several fixed points can be made, but then the correct choice of the position of these points is a significant problem. The way out is to use the POD method, which 
allows one to separate the main spatial characteristics of the flow and obtain their spectral characteristics. On the other hand, in the numerical analysis during verification and comparison it is necessary to take into account the time factor of development or establishment of flows. It can be investigated by comparing the averaged fields for different averaging times. The present work is aimed at the study of these two factors.

\section{Experimental setup}

In the present investigation the experiment was carried out on a benchmark "cube". It is a cubic reservoir with a side of $25 \mathrm{~cm}$ with transparent walls that was filled with water. A constant temperature difference between the lower and upper sides of 20 degrees is maintained with stabilized electrical heating system. This corresponds $\mathrm{Ra}=4.4 \cdot 10^{9}$. The general scheme of the experiment and a separate image taken with a long exposure are shown in Fig. 1.
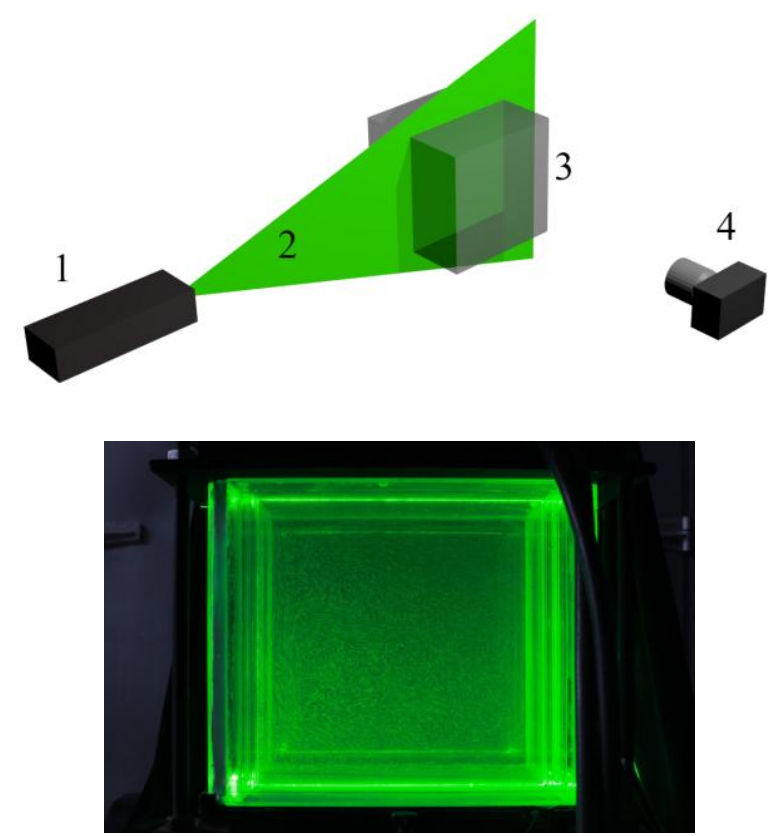

Fig.1. General scheme of the experiment: 1- laser, 2 - laser plane, 3 - benchmark, 4 - camera (top) and separate image taken with a long exposure $(3 \mathrm{sec})$ representing the main flow pattern in system (bottom).

Investigations of the flow structure on the benchmark under conditions of natural convection caused by a vertical temperature gradient are performed using the PIV methods. A low cost PIV-measuring system was used in this study. Continuous diode-pumped green laser LCS-DTL-413 (1.5 W, $527 \mathrm{~nm})$ was used as a source of illumination. The laser plane formed by concave $(2.5$ $\mathrm{mm}$ radius) cylindrical lens intersected the cube vertically. Calibrated HGS particles with a diameter of $10 \mu \mathrm{m}$ were used for seeding the flow. A serial camera Canon EOS 5D Mark II with CMOS 21.1 Mpx sensor and CANON EF $50 \mathrm{~mm} \mathrm{f} / 1.8$ lens (scale $240 \mu \mathrm{m} / \mathrm{px}$ ) was used for recording. It was able to continuously record movies in Full HD $(1920 * 1080)$ format at 29.97 fps. Comparative tests with the Dantec serial system demonstrated that this rather simple system has the ability to resolve the time scales of speed measurement in a wide range: from 0.001 to $30 \mathrm{~Hz}$ for with accuracy less $0.1 \mathrm{~mm} / \mathrm{s}$ for slow flows (velocity does not exceed 2 $\mathrm{cm} / \mathrm{s})$.
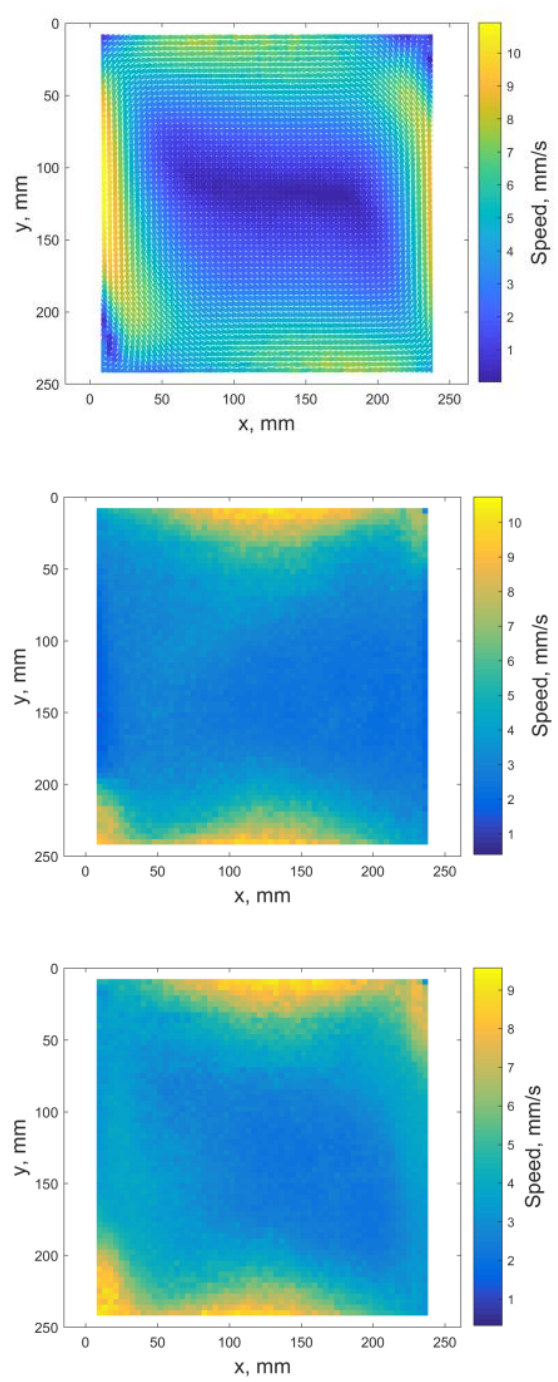

Fig. 2. Average velocity field (top) and a field of standard deviations for horizontal (middle) and vertical (bottom) velocity components.

\section{Data processing}

\subsection{Particle image velocimetry}

A total of 95,928 recording frames were taken, corresponding to 3200 seconds of record duration. Adaptive scheme with Gaussian approximation of the crosscorrelation function was used. PIV processing of the images was performed by specially developed software similar to our previous studies of air velocities in marine boundary layer [7]. Successive frames were compared, interrogation window size $32 \times 32$ px with $50 \%$ overlapping, in one iteration, with tree-point interpolation for $\mathrm{CCF}$ maximum. Velocity fields were filtered using maximum and minimum velocity thresholds. 


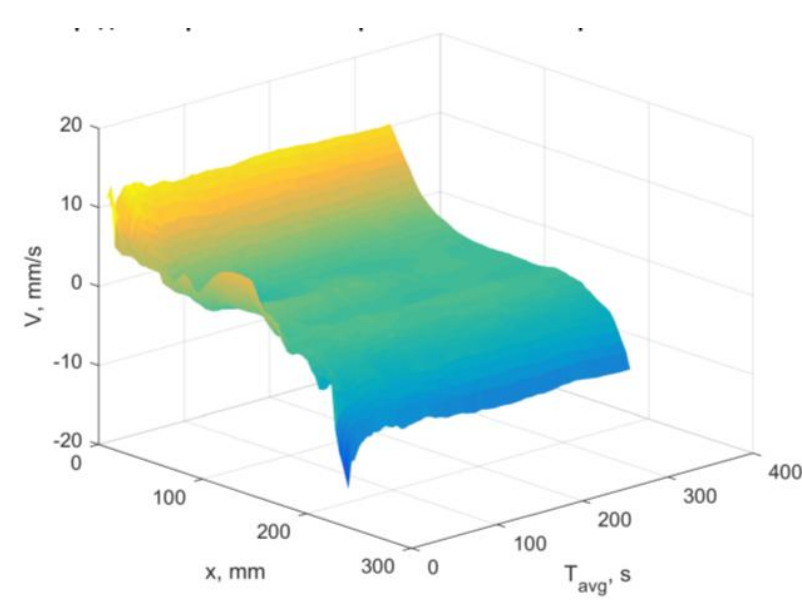

Fig. 3. Dependency of vertical velocity profile on averaging time.

Additional filtering was carried out by 10 -fold downsampling over time. It was made using an averaging with 10 -frame running window. For windows containing less than $30 \%$ of unfiltered velocities, linear time interpolation was performed. As a result, all the measured velocity fields were filtered and merged into a three-dimensional matrix of $61 \times 62 \times 9587$ points.

\subsection{Velocity field analysis}

As mentioned above, the focus of velocity field investigation was on two aspects: the first is the settling time to the flow, and the second is the separating of the characteristic patterns of the flows and their analysis using POD methods.

The obtained average velocity field and fields of velocity fluctuations (see. Fig. 2) were in a good agreement with the results of previous measurements. It was demonstrated that the characteristic settling time is 60 seconds: the dependency of vertical velocity profile on averaging time is shown on Fig. 3; and velocity fields for a set of different averaging times is presented on Fig. 4.

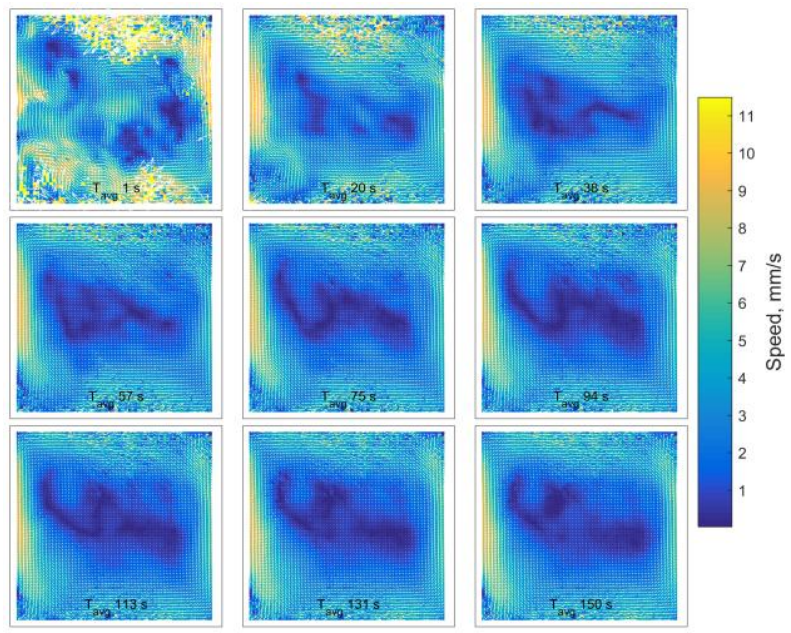

Fig. 4. Velocity fields for a set of 9 different averaging times from 1 to 150 seconds (bottom).

\subsection{Proper orthogonal decomposition}

The high spatial resolution of the obtained velocity fields made it possible to successfully use the Proper orthogonal decomposition (POD) method to isolate the basic spatial modes of the flow.

For the analysis of velocity fields we used the socalled "snapshot POD" technique first suggested by [8]. We used the implementation given in [9]. The use of POD on PIV data has been reported in several publications recently. Examples are [10], who used POD to obtain phase averaged turbulence statistics in a wake flow. In [11] POD based on traditional PIV to interpret short series of time-resolved PIV measurements in a Diesel engine. In [12] POD was used to link details in power spectra to flow structures in an open cavity flow. In [9] POD was used to conjecture three dimensional flow structures and time variation in a jet in crossflow based on traditional PIV measurements.

POD algorithm presents an instantaneous velocity fields as a sum of modes with time-dependant coefficients. In order to calculate them first the fluctuating part of velocity components is arranged for the $\mathrm{N}$ snapshots as:

$$
U=\left[u^{1} u^{2} \ldots u^{N}\right]=\left[\begin{array}{cccc}
u_{1}^{1} & u_{1}^{2} & \cdots & u_{1}^{N} \\
\vdots & \vdots & \vdots & \vdots \\
u_{l m}^{1} & u_{l m}^{2} & \cdots & u_{l m}^{N} \\
v_{1}^{1} & v_{1}^{2} & \cdots & v_{1}^{N} \\
\vdots & \vdots & \vdots & \vdots \\
v_{l m}^{1} & v_{l m}^{2} & \cdots & v_{l m}^{N}
\end{array}\right]
$$

Than the matrix $\tilde{C}=U^{T} U$ is calculated and corresponding eigenvalue problem $\tilde{C} A^{i}=\lambda^{i} A^{i}$ is solved. The solutions are arranged by eigenvalue as: $\lambda_{1}>\lambda_{2}>\ldots>\lambda_{N}=0$. And POD modes are found as:

$$
\phi^{i}=\frac{\sum_{n=1}^{N} A_{n}^{i} u^{n}}{\left\|\sum_{n=1}^{N} A_{n}^{i} u^{n}\right\|}, i=1, \ldots, N
$$

With POD modes arranged as $\Psi=\left[\phi^{1} \phi^{2} \ldots \phi^{N}\right]$ POD coefficients $a_{i}$ can be found for the snapshot $n$ as $a_{i}=\phi^{i} \mathrm{u}^{n}, \mathrm{a}^{n}=\Psi^{T} n^{n}$. A fluctuating part (snapshot) can be reconstructed as:

$$
u^{n}=\sum_{i=1}^{N} a_{i}^{n} \phi^{i}=\Psi \mathrm{a}^{n}
$$

More details on the POD can be found in [13]. The obtained velocity fields were processed with POD method to obtain typical structures (modes) in the flow (Fig. 5). It is shown that the steady flow can be described by combination of the mean flow and the first three modes (up to $95 \%$ of the kinetic energy of the flow is contained in them). It is demonstrated that only starting with the third mode there is a significant change in the spectra of the amplitude coefficients of the modes, the 
peak shifts to the region of higher frequencies to $0.02 \mathrm{~Hz}$ in comparison with the first two modes $(0.006 \mathrm{~Hz})$ (Fig. 6).

Thus accounting for this mode is essential for describing turbulent processes in the system (especially near the walls) and verification of numerical calculations. Comparison with the results of the LES simulation performed using the Ansys CFX package (taking into account the results of processing the experimental data by the POD method) demonstrated good agreement both in the structure of the modes and in their frequency spectra.
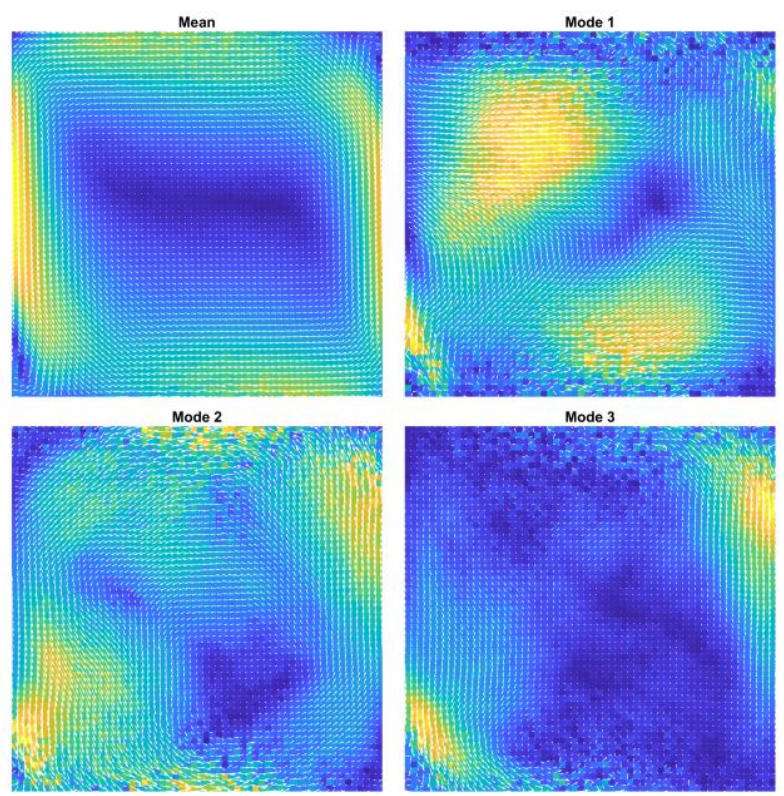

Fig. 5. Mean velocity field and 4 main POD modes, arranged by descending energy.

\section{Conclusion}

Experimental studies of natural convection processes on the benchmark "cube" were performed in the interest of verification problems for CFD processes in power plants, including nuclear ones. The experiments were performed under a constant vertical temperature gradient with a Rayleigh number of $4.4 \cdot 10^{9}$, which corresponds to turbulent flow regimes. PIV system based on the simplest scheme with continuous illumination was used for measurements. For the measured velocity field sequences, the POD method procedure was used to develop the most representative verification methods for numerical CFD calculations. It is shown that more than $95 \%$ of the kinetic energy of turbulent fluctuations is determined by the combination of the first three modes. Thus, a comparison with the results of CFD is reasonable to carry out on the average, as well as on the spectral characteristics of these modes. Studies of the settling processes and characteristic times are also performed.

This work was partial supported by the Russian Foundation of Basic Research No. 18-48-520023 (providing numerical simulations) and Russian Science Foundation No. 18-19-00473 (providing measurements).
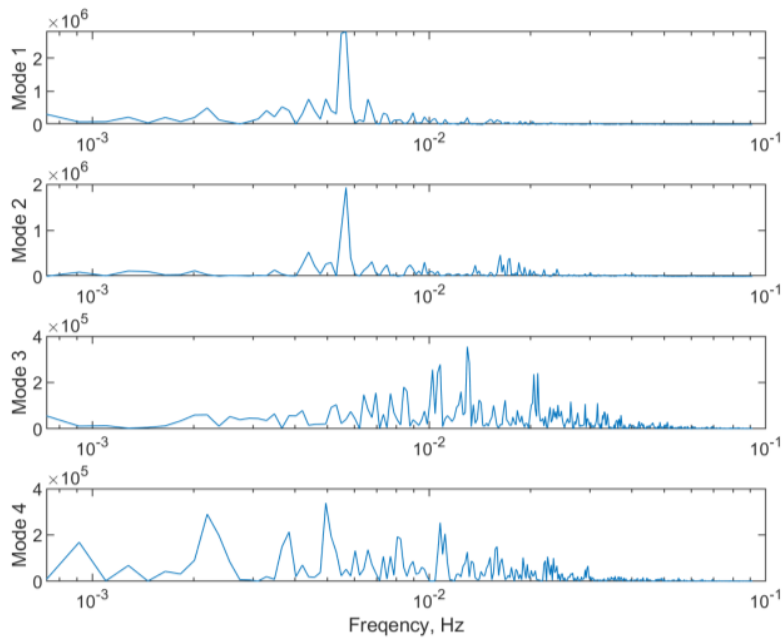

Fig. 6. Spectra of time dependant POD coefficients for 4 main modes.

\section{References}

1. G. Gershun, E. Zhuhovitsky, A. Nepomnyashiy, Stability of convective currents. M.: Science (1989)

2. V. Zimin, P. Frik, Turulent convection, M.: Science (1988).

3. H. Ozoe, K. Yamamoto, S. Churchill, H. Sayama, J. Heat Trans. - T. ASME.. 98 (2), 202, (1976)

4. R. Hernández, R.L. Frederick, Int. J. Heat Mass Tran., 37 (3), 411, (1994)

5. J. Pallarés, I. Cuesta, F.X. Grau, F. Giralt, Int. J. Heat Mass Tran., 39 (15), 3233, (1996)

6. J. Pallarés, F.X. Grau, F. Giralt, Int. J. Heat Mass Tran., 42 (4), 753, (1999)

7. A.A. Kandaurov, Y.I. Troitskaya, D.A. Sergeev, M.I. Vdovin, G.A. Baidakov. Izvestiya, Atmospheric and Oceanic Physics. 50(4), 399(2014)

8. L. Sirovich, Quart. Appl. Math. 45, 561 (1987)

9. K.E. Meyer, J.M. Pedersen, O. Özcan, A turbulent jet in crossflow analysed with proper orthogonal decomposition. J. Fluid Mech. 583, 199 (2007)

10. R. Perrin et. al., Exp. Fluids, 43, 341 (2007)

11. Cosadia, J. Borée, P. Dumont, Diesel engine, Exp. Fluids, 43, 33 (2007).

12. L. Pastur, F. Lusseyran, Y. Fraigneau, B. Podvin, Physical Review E, 72, 065301 (2005)

13. P. Holmes, J. Lumley, G. Berkooz, Turbulence, coherent structures, dynamical systems and symmetry. Cambridge University Press. (1998) 